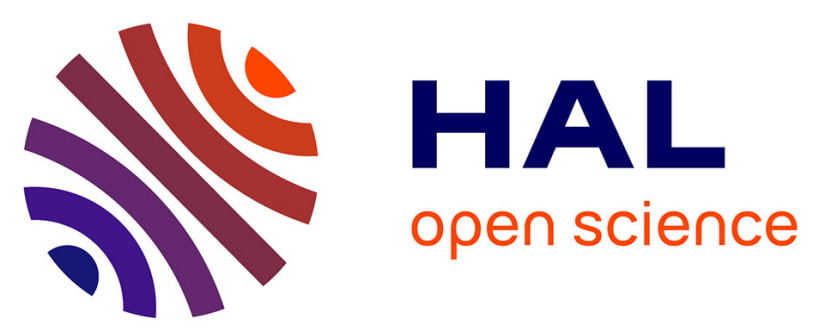

\title{
Characterization and Estimation of the Variations of a Random Convex Set by Its Mean n-Variogram: Application to the Boolean Model
}

\author{
Saïd Rahmani, Jean-Charles Pinoli, Johan Debayle
}

\section{- To cite this version:}

Saïd Rahmani, Jean-Charles Pinoli, Johan Debayle. Characterization and Estimation of the Variations of a Random Convex Set by Its Mean n-Variogram: Application to the Boolean Model. Conférence GSI 2015 - "Geometric Science of Information", École Polytechnique - Université Paris-Saclay; SEE Congress Société de l'Electricité, de l'Electronique et des Technologies de l'Information et de la Communication; THALES AIR SYSTEMS - Technical Directorate Advanced - Department Developments, Oct 2015, Palaiseau, France. pp.296-308, 10.1007/978-3-319-25040-3_33 . emse-01241616

\section{HAL Id: emse-01241616 \\ https://hal-emse.ccsd.cnrs.fr/emse-01241616}

Submitted on 10 Dec 2015

HAL is a multi-disciplinary open access archive for the deposit and dissemination of scientific research documents, whether they are published or not. The documents may come from teaching and research institutions in France or abroad, or from public or private research centers.
L'archive ouverte pluridisciplinaire HAL, est destinée au dépôt et à la diffusion de documents scientifiques de niveau recherche, publiés ou non, émanant des établissements d'enseignement et de recherche français ou étrangers, des laboratoires publics ou privés. 


\title{
Characterization and Estimation of the Variations of a Random Convex Set by its Mean $n$-Variogram : Application to the Boolean Model
}

\author{
Saïd Rahmani, Jean-Charles Pinoli, and Johan Debayle \\ École Nationale Supérieure des Mines de Saint Etienne \\ SPIN/LGF UMR CNRS 5307 \\ said.rahmani@emse.fr, pinoli@emse.fr, debayle@emse.fr
}

Keywords: Boolean model, geometric covariogram, mixed volumes,particle size distribution, $n$ points set probability, random set, shape variations.

\begin{abstract}
In this paper we propose a method to characterize and estimate the variations of a random convex set $\Xi_{0}$ in terms of shape, size and direction. The mean $n$-variogram $\gamma_{\Xi_{0}}^{(n)}:\left(u_{1} \cdots u_{n}\right) \mapsto \mathbb{E}\left[\nu_{d}\left(\Xi_{0} \cap\left(\Xi_{0}-u_{1}\right) \cdots \cap\right.\right.$ $\left.\left.\left(\Xi_{0}-u_{n}\right)\right)\right]$ of a random convex set $\Xi_{0}$ on $\mathbb{R}^{d}$ reveals information on the $n^{\text {th }}$ order structure of $\Xi_{0}$. Especially we will show that considering the mean $n$-variograms of the dilated random sets $\Xi_{0} \oplus r K$ by an homothetic convex family $r K_{r>0}$, it's possible to estimate some characteristic of the $n^{\text {th }}$ order structure of $\Xi_{0}$. If we make a judicious choice of $K$, it provides relevant measures of $\Xi_{0}$. Fortunately the germ-grain model is stable by convex dilatations, furthermore the mean $n$-variogram of the primary grain is estimable in several type of stationary germ-grain models by the so called $n$-points probability function. Here we will only focus on the Boolean model, in the planar case we will show how to estimate the $n^{\text {th }}$ order structure of the random vector composed by the mixed volumes ${ }^{t}\left(A\left(\Xi_{0}\right), W\left(\Xi_{0}, K\right)\right)$ of the primary grain, and we will describe a procedure to do it from a realization of the Boolean model in a bounded window. We will prove that this knowledge for all convex body $K$ is sufficient to fully characterize the so called difference body of the grain $\Xi_{0} \oplus \breve{\Xi}_{0}$. we will be discussing the choice of the element $K$, by choosing a ball, the mixed volumes coincide with the Minkowski's functional of $\Xi_{0}$ therefore we obtain the moments of the random vector composed of the area and perimeter ${ }^{t}\left(A\left(\Xi_{0}\right), U(\Xi)\right)$. By choosing a segment oriented by $\theta$ we obtain estimates for the moments of the random vector composed by the area and the Ferret's diameter in the direction $\theta,{ }^{t}\left(\left(A\left(\Xi_{0}\right), H_{\Xi_{0}}(\theta)\right)\right.$. Finally, we will evaluate the performance of the method on a Boolean model with rectangular grain for the estimation of the second order moments of the random vectors ${ }^{t}\left(A\left(\Xi_{0}\right), U\left(\Xi_{0}\right)\right)$ and ${ }^{t}\left(\left(A\left(\Xi_{0}\right), H_{\Xi_{0}}(\theta)\right)\right.$.
\end{abstract}

\section{Introduction}

A random closed set (RACS) denotes a random variable defined on a probability space $(\Omega, \mathfrak{A}, P)$ taking values in $(\mathbb{F}, \mathfrak{F})$ the family of all closed subset of $\mathbb{R}^{d}$ provided with the $\sigma$-algebra $\mathfrak{F}=\sigma\{\{F \in \mathbb{F} \mid F \cap X \neq \emptyset\} X \in \mathfrak{K}\}$ where $\mathfrak{K}$ denotes the class of compact subsets on $\mathbb{R}^{d}$. As when we work with random vectors it is necessary to give meaning to the concept of distribution. Choquet and Matheron have shown that a random set is fully characterized by its probability of presence in each place of the space, thus the concept of distribution is replaced by the so called functional capacity also called Choquet capacity $T_{\Xi}: \mathfrak{K} \rightarrow[0,1]$.

$$
T_{\Xi}(X)=P(\{\Xi \cap X \neq \emptyset\})
$$

Several materials can be modeled by random sets. In fact, the heterogeneity of the materials can be apprehended by a probabilistic approach [1],[2]. Especially granular or fibrous media [3],[4] can be represented by unions of overlapping particles (the grains) centred on random positions (the germs), thus giving rise to the germ-grain model.

$$
\Xi=\bigcup_{x_{i} \in \Phi} \Xi_{i}+x_{i}
$$

Where $\Phi$ is point process (REF) which generates the germs $x_{i}$, and the grains $\Xi_{i}$ are convex random sets independent and identically distributed. Notice that this definition assumes the independence between the particles $\Xi_{i}$ and their positions $x_{i}$, there is a more general definition authorizing the correlation between germs and grains [5], for more convenience we choose to introduce the model under this hypothesis. There are two types of use of this model. The first one is the simulation of a material, the global characteristics of the model match to the material's ones but the germs and grains have no physical sense: the local characteristics of the model $\left(\Phi\right.$ and $\left.\Xi_{0}\right)$ a priori 
have no connection with the intrinsic structure of the material. Our approach consist in representing the people of crystals by such a model; that is to say that the point process $\Phi$ is the repartition of the particles and the convex random sets $\Xi_{i}$ the particles themselves. The goal is to adjust the model to actual data from measurements acquired by an image acquisition system. Generally, the acquisition is obtained by optical imaging, in other words, we have realizations of $\Xi \cap W$ where $W$ is a bounded window and we want to estimate the characteristics of $\Phi$ and $\Xi_{0}$. To meet this objective, we focuses on two points: first, estimate the characteristics of $\Xi$ from a realization of $\Xi \cap W$ [6], secondly establish relationships between characteristics of $\Xi$ and the local characteristics of the model $\left(\Phi, \Xi_{0}\right)$. This second point raises the problem of non-uniqueness of the representation (2). To remedy this, we introduce an additional assumption: we assume the process $\Phi$ comes from a known type (Poisson process, Cox process, ...). We will use the homogeneous Boolean model, a germ-grain model in which $\Phi$ is a homogeneous Poisson point process. This model is widely used because we have an analytical formula for the Choquet capacity.

$$
T_{\Xi}(X)=1-\exp \left(-\lambda \mathbb{E}\left[\nu_{d}\left(\Xi_{0} \oplus \breve{X}\right)\right]\right), \forall X \in \mathfrak{K}
$$

Several methods are used to connect the global characteristics of the model to the characteristics of the primary grain. In the plane and the space, Miles's formulae [7] or minimum contrast method [8] can estimate the average value of Minkowski's functional of the primary grain. Generally, the primary grain is assumed to have a known and deterministic shape, that is to say, the realizations of the primary grain are homothetic. So as to estimate the variations of the scaling factor from the expectation of the Minkowski functionals. For example, for a disc in the plane, the moments of the first and second orders of the radius of the primary grain are respectively proportional to its average perimeter and its average area. However, if we consider that the shape of the grain can vary, several issues remain unresolved: firstly Minkowski functionals of a random convex set are not enough to characterize its shape and also their average will not provide a sufficient information to characterize its variations. For instance, for a Boolean model whose grain has a shape that depends on several parameters (rectangle, ellipse ...), the estimation of geometric variations of the grain is not direct. The aim of our work is to characterize and estimate the variations of the primary grain of the Boolean model without any assumption concerning its shape, from a realization of the model in a bounded window. The ideal would be to estimate the functional capacity of the primary grain $T_{\Xi_{0}}$ or an equivalent which completely characterizes the random convex $\Xi_{0}$. Applying the Steiner's formula and the linearity of the expectation, for all convex compact set $X$ we have:

$$
\mathbb{E}\left[\nu_{d}\left(\Xi_{0} \oplus \breve{X}\right)\right]=\sum_{k=0}^{d}\left(\begin{array}{l}
d \\
k
\end{array}\right) \mathbb{E}\left[W_{d-k}\left(\Xi_{0}, \breve{X}\right)\right]
$$

Where $W_{d-k}\left(\Xi_{0}, \breve{X}\right)$ denotes the $(d-k)^{t h}$ mixed volume of $\Xi_{0}$ and $\breve{X}$. Considering the relationship (3), we understand that the functional capacity of the model evaluated on a convex $K$ depends on the grain only by the

expectations of mixed volumes between $\Xi_{0}$ and $\breve{X}$. In order to reveal the variations of $\Xi_{0}$ we need to consider the functional capacity of the model on compacts that are not convex, that is why we are interested in the $n$-pointprobability function [5]: $\mathbb{P}\left(\left\{x_{1}, \cdots x_{n}\right\} \subset \Xi\right)$. For $n=2$, this quantity is known under the name of covariance and it can be connected to the mean covariogram of the primary grain [9]. For any $n \geq 3$, the $n$-point-probability function can be used to estimate the mean $n$-variogram $\gamma_{\Xi_{0}}^{(n)}:\left(u_{1} \cdots u_{n}\right) \mapsto \mathbb{E}\left[\nu_{d}\left(\Xi_{0} \cap\left(\Xi_{0}-u_{1}\right) \cdots \cap\left(\Xi_{0}-u_{n}\right)\right)\right]$ of $\Xi_{0}$, this quantity evaluated on the dilated grain $\Xi_{0} \oplus K$ for a convex compact set $K$ reveals the $n^{\text {th }}$ order structure of $\Xi_{0}$, especially some linear combinations of the expectations $\mathbb{E}\left[\prod_{k=0}^{d} W_{d, k}\left(\Xi_{0}, K\right)^{p_{k}}\right]$ of order $p=\sum_{k=0}^{d} p_{k} \leq n$ where $W_{d, k}(\Xi, K), k=0, \cdots d$, denotes the mixed volumes of $\Xi_{0}$ by $K$. First we will discuss the properties of the mean $n$-variogram of a random convex and how it describes its $n^{\text {th }}$ order structure. Secondly we will focus on the planar case, we will discuss the interpretation of the $n^{\text {th }}$ order moments of the vector ${ }^{t}\left(A\left(\Xi_{0}\right), W(\Xi, K)\right)$, and we will show how they can be estimated for the primary grain of a boolean model. Finally we will test the estimation method by the simulations of a boolean model with rectangular grains.

\section{From the Mean $n$-Variogram of a Random Convex Set to its Variations}

In this section we will discuss the properties of the the mean $n$-variogram of a random convex and how it describes his $n^{\text {th }}$ order structure.

\subsection{Mean $n$-Variogram of Random Convex Set}

In this paragraph we will define the mean $n$-variogram of a random convex and discuss its properties. The mean $n$-variogram is a simple generalization of the concept of mean covariogram introduced by Bruno Galerne in [9] for 
$n=2$. The proof of the following results can be easy found by recursion on $n$ from Bruno Galerne's proof [9], that is why we will omit them.

Definition 1. Let $\Xi_{0}$ be a random convex set satisfying $\mathbb{E}\left[\nu_{d}\left(\Xi_{0}\right)\right]<\infty$ and $n \geq 1$ we will call mean n-variogram of $\Xi_{0}$ the expectation of its n-variogram :

$$
\gamma_{\Xi_{0}}^{(n)}: \mid \begin{aligned}
& \mathbb{R}^{d \times(n-1)} \longrightarrow \mathbb{R}_{+} \\
&\left(u_{1}, \cdots u_{n-1}\right) \longmapsto \mathbb{E}\left[\nu_{d}\left(\bigcap_{i=1}^{n-1}\left(\Xi_{0}-u_{i}\right) \cap \Xi_{0}\right)\right]
\end{aligned}
$$

Proposition 1. Let $\Xi_{0}$ be a random convex set satisfying $\mathbb{E}\left[A\left(\Xi_{0}\right)^{n}\right]<\infty$ and $n \geq 3$ (the cases $n<3$ were treated in [9]). Then the mean n-variogram have the following properties:

i) permutation invariant:

$\forall \sigma \in S_{n}, \gamma_{\Xi_{0}}^{(n)}\left(u_{1}, \cdots u_{n-1}\right)=\gamma_{\Xi_{0}}^{(n)}\left(u_{\sigma(1)}, \cdots u_{\sigma(n-1)}\right)$

ii) Reducibility:

$$
\begin{aligned}
& \exists i \neq j, u_{i}=u_{j} \Rightarrow \gamma_{\Xi_{0}}^{(n)}\left(u_{1}, \cdots u_{n-1}\right)=\gamma_{\Xi_{0}}^{(n-1)}\left(u_{1}, \cdots u_{i-1}, u_{i+1}, \cdots u_{n-1}\right) \\
& \text { and } \exists i, u_{i}=0 \Rightarrow \gamma_{\Xi_{0}}^{(n)}\left(u_{1}, \cdots u_{n-1}\right)=\gamma_{\Xi_{0}}^{(n-1)}\left(u_{1}, \cdots u_{i-1}, u_{i+1}, \cdots u_{n-1}\right)
\end{aligned}
$$

iii) $\forall\left(u_{1}, \cdots u_{n-1}\right) \in \mathbb{R}^{d \times(n-1)}, 0 \leq \gamma_{\Xi_{0}}^{(n-1)}\left(u_{1}, \cdots u_{n-1}\right) \leq \gamma_{\Xi_{0}}^{(n-1)}\left(u_{2}, \cdots u_{n-1}\right)$

iv) $\forall k \leq n-1, \int_{\mathbb{R}^{d}} \cdots \int_{\mathbb{R}^{d}} \gamma_{\Xi_{0}}^{(n)}\left(u_{1}, \cdots u_{n-1}\right) d u_{1} \cdots d u_{k}=\ldots$

$\mathbb{E}\left[\nu_{d}\left(\Xi_{0}\right)^{k}\right] \gamma_{\Xi_{0}}^{(n-k)}\left(u_{k+1}, \cdots u_{n-1}\right)$

especially for $k=n$ we have:

$$
\int_{\mathbb{R}^{d}} \cdots \int_{\mathbb{R}^{d}} \gamma_{\Xi_{0}}^{(n)}\left(u_{1}, \cdots u_{n-1}\right) d u_{1} \cdots d u_{n-1}=\mathbb{E}\left[\nu_{d}\left(\Xi_{0}\right)^{n}\right]
$$

v) $\forall\left(u_{1}, \cdots u_{n-1}\right) \in \mathbb{R}^{d \times(n-1)}, \gamma_{\Xi_{0}}^{(n)}\left(-u_{1}, \cdots-u_{n}\right)=\gamma_{-\Xi_{0}}^{(n)}\left(u_{1}, \cdots u_{n}\right)$

and $\gamma_{\Xi_{0}}^{(n)}\left(-u_{1}, u_{2} \cdots u_{n-1}\right)=\gamma_{\Xi_{0}}^{(n)}\left(u_{1}, u_{2}-u_{1}, \cdots u_{n-1}-u_{1}\right)$.

vi) The partial map $u \rightarrow \gamma_{\Xi_{0}}^{(n)}\left(u_{1}, \cdots u_{n-2}, u\right)$ is uniformly continuous and zero limit when $\|u\| \rightarrow+\infty$. Furthermore for all $u \in \mathbb{R}^{d}$ the map

$r \rightarrow \gamma_{\Xi_{0}}^{(n)}\left(u_{1}, \cdots u_{n-2}, r u\right)$ is decreasing on $\mathbb{R}$.

vii) $\gamma_{\Xi_{0}}^{(n)}$ also has an integral formulation:

$$
\gamma_{\Xi_{0}}^{(n)}\left(u_{1}, \cdots u_{n-1}\right)=\int_{\mathbb{R}^{d}} \mathbb{P}\left(\left\{x, x+u_{1}, \cdots x+u_{n-1}\right\} \subset \Xi_{0}\right) d x
$$

The relationship (5) has a the great advantage of giving access to the $n^{\text {th }}$ order moment of the volume of the random set $\Xi_{0}$. This relationship is even more important because dilating $\Xi_{0}$ by a convex $K$, it gives access to some linear combinations of the expectations $\mathbb{E}\left[\prod_{k=0}^{d} W_{d, k}\left(\Xi_{0}, K\right)^{p_{k}}\right]$ of order $p=\sum_{k=0}^{d} p_{k} \leq n$.

\subsection{Dilatation of a Random Convex Set}

Let $\Xi_{0}$ be a convex random set and $K$ a convex compact set, let's recall the Steiner's formula:

$$
\forall r \geq 0, \nu_{d}\left(\Xi_{0} \oplus r K\right)=\sum_{k=0}^{d}\left(\begin{array}{l}
d \\
k
\end{array}\right) W_{d, k}\left(\Xi_{0}, K\right) r^{k}
$$

Where $W_{d, k}\left(\Xi_{0}, K\right)$ denote the mixed volume of $k$-homogeneity in its first variable $(d-k)$-homogeneity in its second, that is:

$$
\forall k=0, \cdots d, \forall(\alpha, \beta) \in \mathbb{R}_{+}^{2}, W_{d, k}\left(\alpha \Xi_{0}, \beta K\right)=\alpha^{d-k} \beta^{k} W_{d, k}\left(\Xi_{0}, K\right)
$$

Furthermore $W_{d, d}(\Xi, K)=\nu_{d}(K)$ and $W_{d, 0}(\Xi, K)=\nu_{d}(\Xi)$. For $r \in \mathbb{R}_{+}, n \geq 0$ and $\Xi_{0}$ satisfiying $\mathbb{E}\left[\nu_{d}\left(\Xi_{0}\right)^{n}\right]<\infty$ we introduce the function:

$$
\zeta_{\Xi_{0}, K}^{(n)}: \mid \begin{aligned}
\mathbb{R}_{+} & \longrightarrow \mathbb{R}_{+} \\
r & \longmapsto \mathbb{E}\left[\nu_{d}\left(\Xi_{0} \oplus r K\right)^{n}\right]
\end{aligned}
$$

The existance of $\zeta_{\Xi_{0}, K}^{(n)}$ is ensured by the existance of $\mathbb{E}\left[\nu_{d}\left(\Xi_{0}\right)^{n}\right]$ and the convexity of $\Xi_{0}$. Notice that for $n \geq 2$ the functional $\zeta_{\Xi_{0}, K}^{(n)}$ can be connected to the mean $n$-variogram of $\Xi_{0} \oplus r K$ by (5) we have: 


$$
\zeta_{\Xi_{0}, K}^{(n)}(r)=\int_{\mathbb{R}^{d}} \cdots \int_{\mathbb{R}^{d}} \gamma_{\Xi \oplus r K}^{(n)}\left(u_{1}, \cdots u_{n-1}\right) d u_{1} \cdots d u_{n-1}
$$

by injecting (7) in (9) we have:

$$
\zeta_{\Xi_{0}, K}^{(n)}(r)=\mathbb{E}\left[\left(\sum_{k=0}^{d}\left(\begin{array}{l}
d \\
k
\end{array}\right) W_{d, k}\left(\Xi_{0}, K\right) r^{k}\right)^{n}\right]
$$

$\zeta_{\Xi_{0}, K}^{(n)}$ is therefore a polynomial function in $r$ of degree $n \times d$ it can be expressed as:

$$
\zeta_{\Xi_{0}, K}^{(n)}=\sum_{j=0}^{n d} C_{n, j}^{(K)} r^{j}
$$

using the multinomial theorem, each of these coefficients $C_{n, j}^{(K)}$ of degree $j \leq n d$, can be expressed as a linear combination of the interactions $\mathbb{E}\left[\prod_{k=0}^{d} W_{d, k}\left(\Xi_{0}, K\right)^{p_{k}}\right]$ satisfying $j=\sum_{k=0}^{d} k \times p_{k}$ and $\sum_{k=0}^{d} p_{k}=n$. Of course we can extract all coefficients $C_{n, j}^{(K)}$ by searching a polynomial approximation of $\zeta_{\Xi_{0}, K}^{(n)}$ in a similar way to the minimum contrast method [10]. Unfortunately in the general case it is not sufficient to obtain the interactions $\mathbb{E}\left[\prod_{k=0}^{d} W_{d, k}\left(\Xi_{0}, K\right)^{p_{k}}\right]$ from the coefficients of $\zeta_{\Xi_{0}, K}^{(n)}$. However in the planar case, we can do this as follows.

Theorem 1. Let $\Xi_{0}$ be a convex random set on the plane $\mathbb{R}^{2}$, we introduce the polynomial function $\eta_{\Xi_{0}, K}^{(n)}$ as follows:

$$
\eta_{\Xi_{0}, K}^{(n)}(r)=\sum_{j=0}^{n}\left(\begin{array}{c}
n \\
j
\end{array}\right)(-1)^{j} A(K)^{j} r^{2 j} \zeta_{\Xi_{0}, K}^{(n-j)}(r)
$$

Where $A(K)=\nu_{2}(K)$ denotes the area of $K$. Then $\eta_{\Xi_{0}, K}^{(n)}$ is a polynomial function of degree $n$ and if we note $M_{n, k}^{(K)}$ its $k^{\text {th }}$ order coefficient for all $n \in \mathbb{N}$ and $k=1, \cdots n$, we have:

$$
M_{n, k}^{(K)}=\sum_{j=0}^{\left\lfloor\frac{k}{2}\right\rfloor}(-1)^{j} A(K)^{j}\left(\begin{array}{l}
n \\
j
\end{array}\right) C_{2 n-2 j, k-2 j}^{(K)}
$$

and

$$
\mathbb{E}\left[A(\Xi)^{n-k} W\left(\Xi_{0}, K\right)^{k}\right]=\frac{M_{n, k}^{(K)}}{2^{k}\left(\begin{array}{l}
n \\
k
\end{array}\right)}
$$

Where $W\left(\Xi_{0}, K\right)=W_{2,1}(K)$ denotes the mixed area and $\left\lfloor\frac{k}{2}\right\rfloor$ denotes the floor of $\frac{k}{2}$.

Proof. First according to Steiner's formula, $A\left(\Xi_{0}\right)+2 r W\left(\Xi_{0}, K\right)=A\left(\Xi_{0} \oplus r K\right)-r^{2} A(K)$

$\Rightarrow \mathbb{E}\left[\left(A\left(\Xi_{0}\right)+2 r W\left(\Xi_{0}, K\right)\right)^{n}\right]=\mathbb{E}\left[\left(A\left(\Xi_{0} \oplus K\right)-r^{2} A(K)\right)^{n}\right]$ by applying the binomial theoerm on each side of the equality and according to linearity of the expectation we have:

$$
\eta_{\Xi_{0}, K}^{(n)}(r)=\sum_{k=0}^{n} 2^{k}\left(\begin{array}{l}
n \\
k
\end{array}\right) r^{k} \mathbb{E}\left[A(\Xi)^{n-k} W\left(\Xi_{0}, K\right)^{k}\right]
$$

it follows the relationship(15). Injecting (12) in (13) we have:

$$
\eta_{\Xi_{0}, K}^{(n)}(r)=\sum_{j=0}^{n} \sum_{p=0}^{2(n-j)}\left(\begin{array}{l}
n \\
j
\end{array}\right)(-1)^{j} A(K)^{j} r^{2 j+p} C_{2 n-2 j, p}^{(K)}
$$

applying the change of variable $z=2 j+p$ we have:

$$
\begin{aligned}
\eta_{\Xi_{0}, K}^{(n)}(r)= & \sum_{j=0}^{n} \sum_{z=2 j}^{2 n}\left(\begin{array}{c}
n \\
j
\end{array}\right)(-1)^{j} A(K)^{j} r^{z} C_{2 n-2 j, z-2 j}^{(K)} \\
& =\sum_{z=0}^{2 n}\left\{\sum_{j=0}^{\left\lfloor\frac{z}{2}\right\rfloor}\left(\begin{array}{c}
n \\
j
\end{array}\right)(-1)^{j} A(K)^{j} C_{2 n-2 j, z-2 j}^{(K)}\right\} r^{z}
\end{aligned}
$$

by identification with (16) it follows the relationship (14). 
Remark 1. The Theorem 1 shows how the $n^{t h}$ order interactions $\mathbb{E}\left[A(\Xi)^{n-k} W\left(\Xi_{0}, K\right)^{k}\right], k=0, \cdots n$, can be estimated by the knowledge of the functions $\zeta_{\Xi_{0}, K}^{(j)}, j=1, \cdots n$. We emphasize that for all convex compact $K$ the distribution of the random vector ${ }^{t}\left(A(\Xi), W\left(\Xi_{0}, K\right)\right)$ is fully characterized and can be reconstructed from its moments: the interactions $\mathbb{E}\left[A(\Xi)^{n-k} W\left(\Xi_{0}, K\right)^{k}\right], n \in \mathbb{N}, k=1, \cdots n$. There is two way to estimate the $n^{\text {th }}$ order interactions: make polynomials approximations of the $\zeta_{\Xi_{0}, K}^{(j)}, j=1, \cdots n$ to get their coefficients $C_{j, p}^{(K)}$ and using (14) or make directly a polynomial approximation of $\eta_{\Xi_{0}, K}^{(n)}$ to get the coefficients $M_{n, k}^{(K)}$ and using (15).

\subsection{Variation of a Random Convex Set in $\mathbb{R}^{2}$}

Here we will discuss the choice of $K$, let $\Xi_{0}$ be a convex random set of $\mathbb{R}^{2}$ satisfying $\mathbb{E}\left[A\left(\Xi_{0}\right)\right]<\infty$. Let's note $B$ the unit ball of $\mathbb{R}^{2}$ and $S_{\theta}$ the rotation of the segment $[0,1] \times\{0\}$ with angle $\theta \in[0,2 \pi]$; in other words $S_{\theta}$ is the centred segment directed by $\theta$ of length two. We have the well know result [11],[6]:

$$
\begin{aligned}
& W\left(\Xi_{0}, B\right)=\frac{1}{2} U\left(\Xi_{0}\right) \\
& W\left(\Xi_{0}, S_{\theta}\right)=H_{\Xi_{0}}(\theta)
\end{aligned}
$$

Where $U\left(\Xi_{0}\right)$ denotes the perimeter of $\Xi_{0}$ and $H_{\Xi_{0}}(\theta)$ denotes the Ferret's diameter of $\Xi_{0}$ in the direction $\theta$. As a direct result, the choice $K=2 B$ provides estimators for all moments of the random vector ${ }^{t}\left(A\left(\Xi_{0}\right), U\left(\Xi_{0}\right)\right)$, and by choosing $K=S_{\theta}$ we obtain all moments of the random vector ${ }^{t}\left(A\left(\Xi_{0}\right), H_{\Xi_{0}}(\theta)\right)$. Notice that the Ferret's diameter is $\pi$-periodic in the variable $\theta$, if $\Xi_{0}$ is supposed to be isotropic, then the random variables $H_{\Xi_{0}}(\theta)$ for $\theta \in[0, \pi]$ are identically distributed. Let's remark that the random process $H_{\Xi_{0}}=\left(H_{\Xi_{0}}(\theta)\right)_{\theta \in[0, \pi]}$ fully characterize the random set $\Xi_{0} \oplus \breve{\Xi}_{0}$; in fact, for each $\omega \in \Omega$ the Ferret's diameter $H_{\Xi_{0}}(\omega):[0, \pi] \rightarrow \mathbb{R}_{+}$coincide with the support function of the convex compact set $\Xi_{0} \oplus \breve{\Xi}_{0}(\omega)$. It is well known that the support function of the convex compact set fully characterizes the convex compact set concerned [12]. Therefore $H_{\Xi_{0}}$ fully characterizes the difference body $\Xi_{0} \oplus \stackrel{\breve{\Xi}}{0}$ that leads to the following theorem.

Theorem 2 (Characterization of a random convex set by its mixed area). Let's $\Xi_{0}^{(1)}$ and $\Xi_{0}^{(2)}$ be two convex random sets of $\mathbb{R}^{2}$ satisfying $\mathbb{E}\left[A\left(\Xi^{(j)}\right)\right]<\infty, j=1,2$ and assume at least one of the distributions of the random variables $A\left(\Xi^{(j)}\right)$ is $M$-determinate [13]. Then, the condition

$$
\forall n \geq 1, \forall K \in \mathfrak{K}_{c}, \zeta_{\Xi_{0}^{(1)}, K}^{(n)}=\zeta_{\Xi_{0}^{(2)}, K}^{(n)}
$$

Implies,

$$
\Xi_{0}^{(1)} \oplus \breve{\Xi}_{0}^{(1)} \stackrel{\mathcal{L}}{=} \Xi_{0}^{(2)} \oplus \breve{\Xi}_{0}^{(2)}
$$

Where "L․

Proof. Let's $\Xi_{0}^{(1)}$ and $\Xi_{0}^{(2)}$ be two convex random sets of $\mathbb{R}^{2}$ satisfying $\mathbb{E}\left[A\left(\Xi_{0}^{(j)}\right)\right]<\infty, j=1,2$ and assume at least one of the distributions of the random variables $A\left(\Xi^{(j)}\right)$ is M-determinate Let's assume the condition (19), according to the Theorem 1 and the M-determinate condition:

$$
\begin{aligned}
(19) & \Rightarrow \forall n \geq 1, \forall K \in \mathfrak{K}, \mathbb{E}\left[W\left(\Xi_{0}^{(1)}, K\right)^{n}\right]=\left[W\left(\Xi_{0}^{(2)}, K\right)^{n}\right] \\
& \Rightarrow W\left(\Xi_{0}^{(1)}, K\right) \stackrel{\mathcal{L}}{=} W\left(\Xi_{0}^{(2)}, K\right)
\end{aligned}
$$

For each $k \geq 1$ and for each $\left(\theta_{1}, \cdots \theta_{k}\right) \in[0, \pi]^{k}$, let $V_{1}={ }^{t}\left(H_{\Xi_{0}^{(1)}}\left(\theta_{1}\right), \cdots H_{\Xi_{0}^{(1)}}\left(\theta_{k}\right)\right)$ be a random vector extract of the random process $H_{\Xi_{1}}$ and $V_{2}=^{t}\left(H_{\Xi_{0}^{(2)}}\left(\theta_{1}\right), \cdots H_{\Xi_{0}^{(2)}}\left(\theta_{k}\right)\right)$ a random vector extract of $H_{\Xi_{0}^{(2)}}$. We will prove $V_{1} \stackrel{\mathcal{L}}{=} V_{2}$, for this, let's consider a positive linear combination of elements of $V_{1}, \sum_{i=1}^{k} \alpha_{i} H_{\Xi_{0}^{(1)}}\left(\theta_{i}\right)$ and the convex compact sets $Z=\bigoplus_{i=1}^{k} \alpha_{i} S_{\theta_{i}}$. Notice the following property:

Lemma 1. Let's $X, Y$ be convex sets, $x \in \mathbb{R}^{+}$and $\beta \in[0, \pi]$. Using the Steiner's formula on $A\left(X \oplus Y \oplus x S_{\beta}\right)$ and the properties of the support function, it is easy to show that:

$$
W\left(X, Y \oplus x S_{\beta}\right)=W(X, Y)+x H_{X}(\beta)
$$


Applaying successively this lemma on $W\left(\Xi_{0}^{(1)}, Z\right)$ and on $W\left(\Xi_{0}^{(2)}, Z\right)$ we have: $W\left(\Xi_{0}^{(1)}, Z\right)=\sum_{i=1}^{k} \alpha_{i} H_{\Xi_{0}^{(1)}}\left(\theta_{i}\right)$ and $W\left(\Xi_{0}^{(2)}, Z\right)=\sum_{i=1}^{k} \alpha_{i} H_{\Xi_{0}^{(2)}}\left(\theta_{i}\right)$

Thus,

$$
\forall\left(\alpha_{1}, \cdots \alpha_{k}\right) \in \mathbb{R}_{+}^{k}, \sum_{i=1}^{k} \alpha_{i} H_{\Xi_{0}^{(1)}}\left(\theta_{i}\right)=\sum_{i=1}^{k} \alpha_{i} H_{\Xi_{0}^{(2)}}\left(\theta_{i}\right)
$$

which implies $V_{1} \stackrel{\mathcal{L}}{=} V_{2}$, we have this result for all $k \geq 1$ and for all $\left(\theta_{1}, \cdots \theta_{k}\right) \in[0, \pi]^{k}$, thus $H_{\Xi_{0}^{(1)}} \stackrel{\mathcal{L}}{=} H_{\Xi_{0}^{(2)}}$ therefore $\Xi_{0}^{(1)} \oplus \breve{\Xi}_{0}^{(1)} \stackrel{\mathcal{L}}{=} \Xi_{0}^{(2)} \oplus \breve{\Xi}_{0}^{(2)}$.

Remark 2. First note that the M-determinate condition is not realy restrictive, it can always be assumed in pratical cases [14]. Let's notice that the choice of the convex compact $Z=\bigoplus_{i=1}^{k} \alpha_{i} S_{\theta_{i}}$ provide an explicit expression of the mixed area, it can be use for estimate all characteristics of the random process $H_{\Xi_{0}}$ in the following way; For each $k \geq 1$ and $n \geq 0$ we define $P_{\Xi_{0},\left(\theta_{1}, \cdots \theta_{k}\right)}^{(k, n)}$, the polynomial function on $k$ variables of degree $n$ as:

$$
\begin{aligned}
P_{\Xi_{0},\left(\theta_{1}, \cdots \theta_{k}\right)}^{(k, n)}\left(\alpha_{1}, \cdots \alpha_{k}\right) & =\mathbb{E}\left[W\left(\Xi_{0}, \bigoplus_{i=1}^{k} \alpha_{i} S_{\theta_{i}}\right)^{n}\right] \\
& =\left(\sum_{i=1}^{k} \alpha_{i} H_{\Xi_{0}}\left(\theta_{i}\right)\right)^{n} \\
& =\sum_{j_{1}+\cdots j_{k}=n}\left(\begin{array}{c}
n \\
j_{1}, \cdots j_{k}
\end{array}\right) \mathbb{E}\left[\prod_{i=1}^{k} H_{\Xi_{0}}\left(\theta_{i}\right)^{j_{i}}\right] \prod_{i=1}^{k} \alpha_{i}^{j_{i}}
\end{aligned}
$$

Thus all of the expectations $\mathbb{E}\left[\prod_{i=1}^{k} H_{\Xi_{0}}\left(\theta_{i}\right)^{j_{i}}\right]$ can be estimate by a fit of $P_{\Xi_{0},\left(\theta_{1}, \cdots \theta_{k}\right)}^{(k, n)}$. However, in practice it is difficult to compute and fit $P_{\Xi_{0},\left(\theta_{1}, \cdots \theta_{k}\right)}^{(k, n)}$ for large $k$, thus we will be more interested in the autocorrelation of the random process $H_{\Xi_{0}}$ and its marginals moments $\mathbb{E}\left[H_{\Xi_{0}}(\theta)^{n}\right]$.

\section{Application to the Boolean Model}

Let $\Xi$ be a Boolean of primary grain $\Xi_{0}$ and intensity $\lambda$ :

$$
\Xi=\bigcup_{x_{i} \in \Phi} \Xi_{i}+x_{i}
$$

Where $\Phi$ is a homogeneous Poisson point process of intensity $\lambda$ and the $\Xi_{i}$ are random convex sets identically distributed as $\Xi_{0}$.

\subsection{The Polynomial $\zeta_{\Xi_{0}}^{(K)}$ for the Primary Grain of the Boolean Model}

Let's recall the fundamental relationship of functional capacity:

$$
\begin{aligned}
\forall X \in \mathfrak{K}, T_{\Xi}(X) & =P(\{\Xi \cap X \neq \emptyset\}) \\
& =1-\exp \left(-\lambda \mathbb{E}\left[\nu_{d}\left(\Xi_{0} \oplus \breve{X}\right)\right]\right) \\
& =1-\exp \left(-\Psi_{\Xi}(X)\right)
\end{aligned}
$$

Where $\Psi_{\Xi}(X)=-\ln \left(1-T_{\Xi}(X)\right)=\lambda \mathbb{E}\left[\nu_{d}\left(\Xi_{0} \oplus \breve{X}\right)\right]$. Let's enunciate a useful lemma the proof is omitted since it can be established by induction.

Lemma 2 (inclusion-exclusion principle). Let $f$ be a $C$-additive sets function, and $\left(A_{i}\right)_{1 \leq i \leq n}$ be a non degenerate family of subset of $\mathbb{R}^{n}$, then:

$$
\begin{aligned}
& f\left(\bigcap_{i=1}^{n} A_{i}\right)=\sum_{k=1}^{n} \frac{(-1)^{k+1}}{k !} \sum_{\left(i_{1}, \cdots i_{k}\right) \in I_{k}} f\left(\bigcup_{j=1}^{k} A_{i_{j}}\right) \\
& f\left(\bigcup_{i=1}^{n} A_{i}\right)=\sum_{k=1}^{n} \frac{(-1)^{k+1}}{k !} \sum_{\left(i_{1}, \cdots i_{k}\right) \in I_{k}} f\left(\bigcap_{j=1}^{k} A_{i_{j}}\right)
\end{aligned}
$$

Where $I_{k}=\left\{\left(i_{1}, \cdots i_{k}\right) \in\left\{i_{1}, \cdots i_{k}\right\} \subset\{1, \cdots n\}^{k} \mid \forall l \leq k, \forall m \leq k, l \neq m \Rightarrow i_{l} \neq i_{m}\right\}$. 
For $n \geq 2$ let's note $u_{n}=0$, according the Lemma 2 and the expression of $\Psi$, the mean $n$-variogram can be expressed as:

$$
\begin{aligned}
\gamma_{\Xi_{0}}^{(n)}\left(u_{1}, \cdots u_{n-1}\right) & =\mathbb{E}\left[\nu_{d}\left(\bigcap_{i=1}^{n} \Xi_{0}-u_{i}\right)\right] \\
& =\sum_{k=1}^{n} \frac{(-1)^{k+1}}{k !} \sum_{(i(1), \cdots i(k)) \in I_{k}} \mathbb{E}\left[\nu_{d}\left(\bigcup_{j=1}^{k} \Xi_{0}-u_{i(j)}\right)\right] \\
& =\sum_{k=1}^{n} \frac{(-1)^{k+1}}{\lambda k !} \sum_{(i(1), \cdots i(k)) \in I_{k}} \Psi_{\Xi}\left(\left\{u_{i(1)}, \cdots u_{i(k)}\right\}\right) \\
\Rightarrow \gamma_{\Xi_{0}}^{(n)}\left(u_{1}, \cdots u_{n-1}\right) & =\sum_{k=1}^{n} \frac{(-1)^{k}}{\lambda k !} \sum_{(i(1), \cdots i(k)) \in I_{k}} \ln \left(1-T_{\Xi}\left(\left\{u_{i(1)}, \cdots u_{i(k)}\right\}\right)\right)
\end{aligned}
$$

Obviously the quantities $\Psi_{\Xi}\left(\left\{u_{i_{1}}, \cdots u_{i_{k}}\right\}\right)$ can be expressed by the $n$-point probability function $\mathscr{C}_{\Xi}^{(n)}\left(x_{1}, \cdots x_{n}\right)=$ $\mathbb{P}\left(\left\{x_{1}, \cdots x_{n}\right\} \subset \Xi\right)$ evaluated on the subsets of $\left\{u_{1}, \cdots u_{n}\right\}$, we have:

$$
\begin{aligned}
& \ln \left(1-T_{\Xi}\left(\left\{u_{i_{1}}, \cdots u_{i_{k}}\right\}\right)\right)=\ln \left(\mathbb{P}\left(\bigcap_{j=1}^{k}\left\{u_{i_{j}} \notin \Xi\right\}\right)\right) \\
&=\ln \left(\sum_{j=1}^{k} \frac{(-1)^{j+1}}{j !} \sum_{\left(z_{1}, \cdots z_{j}\right) \in I_{j}} \mathbb{P}\left(\bigcup_{l=1}^{j}\left\{u_{i\left(z_{l}\right)} \notin \Xi\right\}\right)\right) \\
&=\ln \left(\sum_{j=1}^{k} \frac{(-1)^{j+1}}{j !} \sum_{\left(z_{1}, \cdots z_{j}\right) \in I_{j}}\left(1-\mathscr{C}_{\Xi}^{(j)}\left(u_{i\left(z_{1}\right)}, \cdots u_{i\left(z_{j}\right)}\right)\right)\right. \\
& \Rightarrow \gamma_{\Xi_{0}}^{(n)}\left(u_{1}, \cdots u_{n-1}\right)=\sum_{k=1}^{n} \sum_{(i(1), \cdots i(k)) \in I_{k}} \frac{(-1)^{k}}{\lambda k !} \ln \left(\sum_{j=1}^{k} \frac{(-1)^{j+1}}{j !} \sum_{\left(z_{1}, \cdots z_{j}\right) \in I_{j}}\left(1-\mathscr{C}_{\Xi}^{(j)}\left(u_{i\left(z_{1}\right)}, \cdots u_{i\left(z_{j}\right)}\right)\right)\right.
\end{aligned}
$$

The $n$-point-probability function $\mathscr{C}_{\Xi}^{(n)}\left(x_{1}, \cdots x_{n}\right)$ can be viewed as a volume fraction of $\bigcap_{i=1}^{n}\left(\Xi+x_{i}\right)$, it is easy to see that the stationary of $\Xi$ implies $\mathbb{P}\left(\left\{x_{1}, \cdots x_{n}\right\} \subset \Xi\right)=\mathbb{P}\left(\left\{0, x_{1}-x_{n}, \cdots x_{n-1}-x_{n}\right\} \subset \Xi\right)$,its yield an unbiased estimator for the $n$-point probability function in bounded windows $W$ :

$$
\hat{\mathscr{C}}_{\Xi, W}^{(n)}\left(x_{1}, \cdots x_{n}\right)=\frac{\nu_{d}\left((\Xi \cap W) \ominus\left\{0, x_{1}-x_{n}, \cdots x_{n-1}-x_{n}\right\}\right)}{\nu_{d}\left(W \ominus\left\{0, x_{1}-x_{n}, \cdots x_{n-1}-x_{n}\right\}\right)}
$$

Thus, an estimator $\hat{\gamma}_{\Xi_{0}, W}^{(n)}$ for the mean $n$-variogram of $\Xi_{0}$ can be obtained by (25),(24) associated to an estimator of $\lambda$ (see [7],[5]). We emphasize that the Boolean model is stable by convex dilatation, in other words, for $K \in \mathfrak{K}_{c}$, the dilated model $\Xi \oplus K$ is also a Boolean model of same intensity $\lambda$ and of primary grain $\Xi_{0} \oplus K$. As a consequence, for each $r \geq 0$ an estimator $\hat{\gamma}_{\Xi_{0} \oplus r K}^{(n)}$ can be found. However a precaution must be taken to break the edge effects; if we have a realization of $\Xi \cap W$, the dilated model $\Xi \oplus r K$ is only known within the eroded window $W_{r K}=W \ominus r K$, in fact $((\Xi \cap W) \oplus r K) \cap W_{r K}=(\Xi \oplus r K) \cap W_{r K}$. Therefore it follow from (10) the estimators:

$$
\hat{\zeta}_{\Xi_{0}, K, W}^{(n)}(r)=\int_{\mathbb{R}^{d}} \cdots \int_{\mathbb{R}^{d}} \hat{\gamma}_{\Xi \oplus r K, W_{r K}}^{(n)}\left(u_{1}, \cdots u_{n-1}\right) d u_{1} \cdots d u_{n-1}
$$

\subsection{The Case of the Planar Boolean Model}

As a consequence of $(26)$ and the Theorem 1, in the planar case we obtain an estimator for the polynomial $\eta_{\Xi, K}^{(n)}$ :

$$
\hat{\eta}_{\Xi_{0}, K, W}^{(n)}(r)=\sum_{j=0}^{n}\left(\begin{array}{c}
n \\
j
\end{array}\right)(-1)^{j} A(K)^{j} r^{2 j} \hat{\zeta}_{\Xi_{0}, K, W}^{(n-j)}(r)
$$


Therefore by fitting these quantities, we obtain estimators for the moments of ${ }^{t}\left(A\left(\Xi_{0}\right), W\left(\Xi_{0}, K\right)\right)$. Furthermore the polynomial approximation of $\hat{\eta}_{\Xi_{0}, K, W}^{(n)}$ can be refined by inequality constraints, some of them are probabilistic( inequality between moments, Cauchy-Schwarz inequality). But there are also some morphological constraints like the generalized isoperimetric inequality [15]. Furthermore, if we make additive assumptions concerning the shape of $\Xi_{0}$, other constraints can be found [16].

We have tested this method with $n=2$ for a Boolean model of rectangular grains: the uncorrelated sides of the primary grain follow $\mathcal{N}(40,10)$ and $\mathcal{N}(30,10)$, the intensity parameter $\lambda=\frac{100}{500 \times 500}$ (see Figure 1 ). We have simulated several realization of this model in a bounded window $W=500 \times 500$, and we studies the relative error between the theoretical and estimated moments of ${ }^{t}\left(A\left(\Xi_{0}\right), W\left(\Xi_{0}, K\right)\right)$ when $K$ is a ball or a segment (see Figure 1 ).

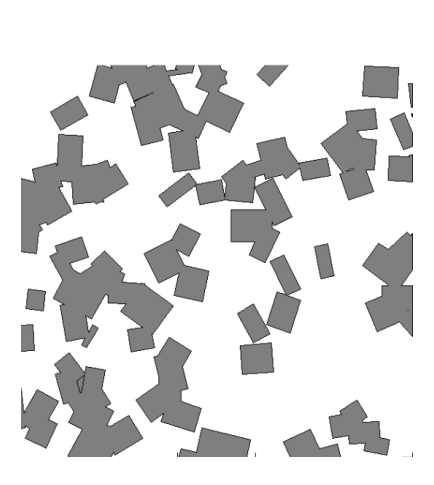

(a)

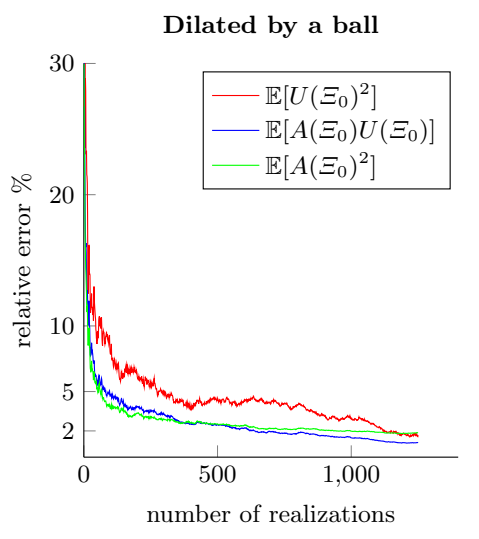

(b)

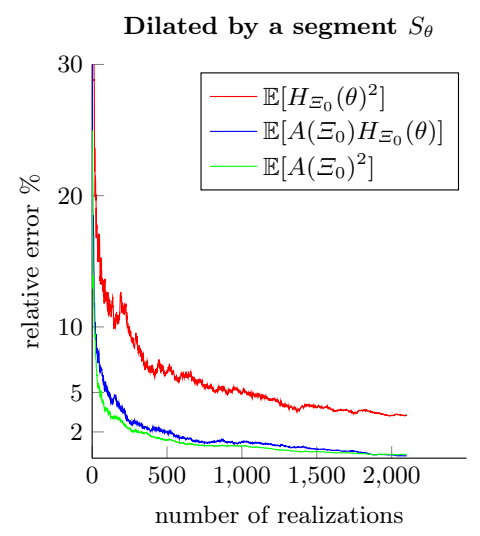

(c)

Fig. 1: A realization of the test model (a) and the relatives error of the estimation of the $2^{\text {nd }}$ order moments of ${ }^{t}\left(A\left(\Xi_{0}\right), U\left(\Xi_{0}\right)\right)$ on (b) and of ${ }^{t}\left(A\left(\Xi_{0}\right), H_{\Xi_{0}}(\theta)\right)$ on (c).

\section{Conclusions and Prospects}

We have established analytical formulae which allow us to connect the $n$-variograms of the dilatations of a random convex set with the variations of some of its morphological characteristics. For the Boolean model, we also have shown how the $n$-variogram of its primary grain can be connected to its $n$-point probability function. Therefore, it provides estimators of the variations of the primary grain's morphological characteristics. Especially, using dilatation by a disk or a segment, the proposed method can be used to characterize a primary grain whose shape depends on two parameters (rectangle, ellipse,...). We emphasize that our method can be used for any germ-grain model in which we can estimate the mean $n$-variogram.

In the future we are looking at more complex germ-grain models than the Boolean model. We are also interested in the influence of the model parameters and the observation's window on the accuracy of the estimates. The prospect of describing a convex random set by the characteristics of the random process associated to its Ferret's diameter (see subsection 2.3), is even more relevant.

\section{References}

1. S Torquato. Random heterogeneous materials: microstructure and macroscopic properties, volume 16 . Springer Science \& Business Media, 2002.

2. D. Jeulin. Random texture models for material structures. Statistics and Computing, 10(2):121-132, 2000.

3. B Galerne. Modèles d'image aléatoires et synthèse de texture. PhD thesis, Ecole normale supérieure de Cachan-ENS Cachan, 2010.

4. C Peyrega. Prediction des proprietes acoustiques de materiaux fibreux heterogenes a partir de leur microstructure 3D. $\mathrm{PhD}$ thesis, École Nationale Supérieure des Mines de Paris, 2010.

5. S.N Chiu, D. Stoyan, W.S. Kendall, and J. Mecke. Stochastic geometry and its applications. John Wiley \& Sons, 2013.

6. I. S. Molchanov. Statistics of the Boolean model for practitioners and mathematicians. Wiley Chichester, 1997. 
7. R.E. Miles. Estimating aggregate and overall characteristics from thick sections by transmission microscopy. Journal of Microscopy, 107(3):227-233, 1976.

8. D. Stoyan. Stochastic Geometry and its application. John Wiley \& Sons, 1987.

9. B. Galerne. Computation of the perimeter of measurable sets via their covariogram. applications to random sets. Image Analysis and Stereology, 30(1):39-51, 2011.

10. L. Heinrich. Asymptotic properties of minimum contrast estimators for parameters of boolean models. Metrika, 40(1):6794, 1993.

11. K. Michielsen and H. De Raedt. Integral-geometry morphological image analysis. Physics Reports, 347(6):461-538, 2001.

12. R. Schneider. Convex bodies: the Brunn-Minkowski theory, volume 151. Cambridge University Press, 2013.

13. J. Stoyanov. Krein condition. in Hazewinkel, Michiel, Encyclopedia of Mathematics, Springer, ISBN 978-1-55608-010-4, 2001.

14. Akhiezer $\mathrm{N}$ and Kemmer N. The classical moment problem: and some related questions in analysis, volume 5 . Oliver \& Boyd Edinburgh, 1965.

15. Shing-Tung Yau and NJ) Institute for Advanced Study (Princeton. Seminar on differential geometry. Number 102. Princeton University Press, 1982.

16. S. Rivollier, J. Debayle, and J-C. Pinoli. Analyse morphométrique d'images à tons de gris par diagrammes de forme. In RFIA 2012 (Reconnaissance des Formes et Intelligence Artificielle), pages 978-2, 2012. 